\title{
A special issue on the renin-angiotensin system
}

\author{
Hayo Castrop • Armin Kurtz • Frank Schweda
}

Published online: 14 November 2012

(C) Springer-Verlag Berlin Heidelberg 2012

The first elements of an endocrine pressor system, today known as the renin-angiotensin system, were discovered some 100 years ago. Soon after its discovery, the clinical relevance of the renin-angiotensin system became apparent, and since then, intense research has continuously uncovered new facets of the diverse functions of the renin-angiotensin system. Classically considered an endocrine system that regulates blood pressure and volume homoeostasis, the spectrum of functions of the renin-angiotensin system has been expanded to include several non-hemodynamic elements, including prohypertrophic and pro-fibrotic effects, among others. These novel aspects of the renin-angiotensin system are generally more apparent under pathophysiological conditions, but they may also be relevant during normal growth and differentiation.

Several recent reviews have covered a broad range of aspects regarding the regulation and function of the systemic and the newly discovered local renin-angiotensin systems $[1,2]$.

With Pfluger's Special Issue on the renin-angiotensin system, we have attempted to cover the key elements that account for the regulation of the renin-angiotensin system and address the most important aspects of its functional output. Furthermore, compared to the majority of recent reviews, we aimed to include new trends and developments, some of which are still controversial and preliminary, but all of which have what we consider a high potential for research progress in the next years. The articles of this Special Issue were assembled with

H. Castrop $(\bowtie) \cdot$ A. Kurtz $\cdot$ F. Schweda

Institute of Physiology, University of Regensburg,

Regensburg, Germany

e-mail: hayo@castrop.com the goal of ensuring that even the most highly specialized contributions would be informative and relevant for the broad readership of our journal.

The articles of the Special Issue encompass the entire cascade of the renin-angiotensin system, starting with the substrate angiotensinogen, moving on to the control of renin expression and secretion as the rate-limiting step of the cascade, and eventually addressing the effectors of the renin-angiotensin system, such as the classical angiotensin II AT receptors, the newly discovered angiotensin receptor-associated proteins, the highly controversial (pro)renin receptor, and receptors for alternative derivatives such as angiotensin (1-7), for which the biological relevance is just beginning to be uncovered. Further contributions address new aspects of the local renin-angiotensin systems, like the intra-renal and the brain renin-angiotensin system. The Special Issue concludes with articles that integrate the renin-angiotensin system into a broader context, such as the regulation of body fluid composition and the regulation of metabolic rate. Given the clinical relevance of the renin-angiotensin system, these more integrative articles are particularly focused on data from humans.

We thank all the experts in the field who contributed to this Special Issue-it is your time and commitment that have made this collection of articles a success.

\section{References}

1. Castrop H, Hocherl K, Kurtz A, Schweda F, Todorov V, Wagner C (2010) Physiology of kidney renin. Physiol Rev 90:607-673

2. Paul M, Poyan Mehr A, Kreutz R (2006) Physiology of local renin-angiotensin systems. Physiol Rev 86:747-803 